\title{
The Algorithm of Spectrum Detection Based on Signal Sampling Autocorrelation
}

\author{
Jianjun $\mathrm{Tao}^{1}$, Xiaoqin $\mathrm{Wu}^{1+}$ \\ ${ }^{1}$ College of Information Science and Technology, Hainan Universtiy, Haikou,Hainan,China
}

\begin{abstract}
Spectrum sensing is the key technology of cognitive radio. According to the statistical characteristics of signal and noise we can determine whether the received signal contains the primary user signal. In this paper, we have a deep study on spectrum sensing algorithms based on signal autocorrelation. Study on the influence of various parameters on threshold and the relationship between threshold and detection probability are derived. Through the combination of theoretical simulation and experimental signal simulation, we have an overall explanation of the performance of the detection algorithm based on signal autocorrelation. The influence of different channels on the detection performance is also studied. Comparing with the energy detection algorithm, the algorithm in this paper has good robustness.
\end{abstract}

Keywords: Spectrum sensing, Sampling autocorrelation, Energy detection

\section{Introduction}

With the rapid development of wireless communication, fixed spectrum-allocaton policies lead to low spectrum usage im many frequency bands. An effective way to solve the problem of low spectrum utilization and spectrum scarcity is to use spectrum sensing technology to detect the spectrum idle frequency and then carry on dynamic spectrum access. In the development of cognitive radio technology, a variety of spectrum sensing methods appeared. Mainly includes early energy detection[1], matching filter detection[2], cyclostationary feature detection [3], as well as the later methods: the detection based on random matrix theory [4] etc. In the case that the information of primary user signal cannot be obtained, energy detection algorith is simple and has low computational cost, so it is the optimal detection algorithm. However, its performance is affected by the noise uncertainty so that the robustness of this algorithm is not strong[5]. For the past several years, the spectrum sensing algorithms based on random matrix theory are proposed[6][7]. According to the special properties of the empirical spectrum distribution function of signal and noise, the effect of noise uncertainty on spectrum sensing can be avoided. The literature[8] proposed the MME detection, utilized the distribution law of the maximum eigenvalue, optimized the self-sensing properties of the system in the case of finite samples. However, the distribution function of this algorithm is not clear, only specific values can be obtained by lookup table. Yonghong Zeng proposed the algorithms based on covariance absolute value detection (CAV) [9][10], the algorithms utilized the different characteristics of the autocorrelation matrix of the primary user signal and noise to determine whether the primary user signal exists. The detection is a kind of blind detection so that no prior information of primary user signal is required. At the same time, it is insensitivity to noise uncertainty. The literature [11] according to simulation analysis of CAV detection algorithm for rayleigh fading channel, but the detection performance of this algorithm is not comprehensive enough.

In this paper, we made depth analysis on the autocorrelation matrix detection algorithm, studied the influence of various parameters on threshold, deserved the relationship between threshold and detection

+ Corresponding author. Tel.: + (0898)66279279.

E-mail address: xq_wu@hainu.edu.cn. 
probability and this relationship can be further used for the detection of stepping broadband spectrum detection. And also we analysed the influence of different channels on the detection performance. Through the simulation of the theoretical derivation and experimental signals, we can have an overall explanation of the performance of the detection algorithm based on autocorrelation matrix.

\section{System Modeling}

\subsection{System Structure and Signal Models}

Let $y(t)=s(t)+\eta(t)$ be the continuous-time received signal, where $s(t)$ is the possible primary user's signal and $\eta(t)$ is the noise. Let $f_{s}$ be the sampling rate, and $N$ be the number of the received samples. After sampling, the obtained samples are denoted by $y(n), n=1, \ldots, N-1$, the signal detection can be formulated as a binary hypotheses testing problem as follows

$$
\begin{array}{ll}
H_{0}: & y(n)=\eta(n) \\
H_{1}: & y(n)=h \cdot s(n)+\eta(n), n=0, \ldots, N-1
\end{array}
$$

where $H_{0}$ and $H_{1}$ represent the hypothesis "primary signal absent" and the hypothesis "primary signal present", respectively. $\eta(n)$ is the noise and is assumed to be complex AWGN with zero mean and variance $\sigma^{2}, s(n)$ is the primary signal, which can be any complex signal. $N$ is the total number of samples collected in the sensing time $T ; h$ denote the channel fading process. Without loss of generality, assume $s(n)$ and $\eta(n)$ are both independent and real signals. The main performace specifications spectrum sensing are the probability of false alarm $\left(P_{f a}\right)$ and the probability of detection $\left(P_{d}\right)$. For a good detection algorithm, $P_{d}$ should be high, and $P_{f a}$ should be low. Usually, we choose the threshold based on $P_{f a}$. Based on IEEE802.22 document we can know that the reference range are given by

$$
P_{f a}=0.01 \sim 0.1, P_{d}=0.9 \sim 0.99 .
$$

\subsection{Construction of Detection Statistics}

For the convenience of discussion, the received signal under the $H_{1}$ situation can be rewritten as $y(n)=m(n)+\eta(n)$, where $m(n)$ is primary user signal which passes a wireless communication channel. Consider $L$ consecutive samples and define the following vectors:

$$
\begin{aligned}
& y(n)=\left[\begin{array}{llll}
y(n) & y(n-1) & \cdots & y(n-L+1)
\end{array}\right]^{T} \\
& m(n)=\left[\begin{array}{llll}
m(n) & m(n-1) & \cdots & m(n-L+1)
\end{array}\right]^{T} \\
& \eta(n)=\left[\begin{array}{llll}
\eta(n) & \eta(n-1) & \cdots & \eta(n-L+1)
\end{array}\right]^{T}
\end{aligned}
$$

Where $L$ is called the smoothing factor.

The statistical covariance matrices of the signal and noise are defined as

$$
\begin{aligned}
R_{y} & =E\left[y(n) y(n)^{T}\right] \\
R_{m} & =E\left[m(n) m(n)^{T}\right]
\end{aligned}
$$

We can verify that

$$
R_{y}=R_{m}+R_{\eta}
$$

And because of the randomness and irrelevance of noise, we can easily verity that

$$
R_{y}=R_{m}+\sigma_{\eta}^{2} I_{L}
$$

So, if there is a signal and the signal samples are related, then $R_{m}$ is not a diagonal matrix. Otherwise, the off-diagonal elements of $R_{y}$ is all zeros. Denote $r_{i j}$ as the element of matrix $R_{y}$ at the $i$ th row and $j$ th column, $r_{i i}$ is the diagonal elements of $R_{y}$. Let $T_{1}$ be the average of all $r_{i j}$ and $T_{2}$ be the average of all $r_{i i}$, we define that

$$
\begin{aligned}
& T_{1}=\frac{1}{L} \sum_{i=1}^{L} \sum_{j=1}^{L}\left|r_{i j}\right| \\
& T_{2}=\frac{1}{L} \sum_{i=1}^{L}\left|r_{i i}\right|
\end{aligned}
$$


Then we can detect the presence of the signal according to the $T_{1} / T_{2}$. when $T_{1} / T_{2}>1$, the primary user signal is present, when $T_{1} / T_{2}=1$, no primary user signal. But in practice, statistical covariance matrices can only be computed using a limited number of signal samples. In this paper, we calculate and estimate the sample autocorrelation by limited number of received signals as follows

$$
\lambda(l)=\frac{1}{N_{s}} \sum_{m=0}^{N_{s}-1} y(m) y(m+l), \quad l=0,1, \cdots, L-1
$$

where $N_{s}$ is the number of sampling points. We can prove that if $N_{s} \rightarrow \infty$ and $l<<N_{s}$, the autocorrelation matrix is approximated given by

$$
R_{y}\left(N_{s}\right)=\left(\begin{array}{cccc}
\lambda(0) & \lambda(1) & \cdots & \lambda(L-1) \\
\lambda(1) & \lambda(0) & \cdots & \lambda(L-2) \\
\vdots & \vdots & \ddots & \vdots \\
\lambda(L-1) & \lambda(L-2) & \cdots & \lambda(0)
\end{array}\right)_{L \times L}
$$

Now, the sample covariance matrix is a toeplitz and symmetric matrix. According to (9) and (10), we can calculate the value of $T_{1} / T_{2}$. However, noise can not be as good as theoretical value, that is only the diagonal elements are zeros. Therefore, a modified threshold value $\gamma$ is needed to compare with $T_{1} / T_{2}$. The threshold is usually calculated based on a certain false alarm probability. According to the calculation of Yonghong Zeng in literature [8], $\gamma$ can be expressed as

$$
\gamma=\frac{\left.1+(L-1) \sqrt{\frac{2}{N_{s} \pi}}\right)}{1-Q^{-1}\left(P_{f a}\right) \sqrt{\frac{2}{N_{s}}}}
$$

When $T_{1} / T_{2}>\gamma$, the primary signal is present; when $T_{1} / T_{2}<\gamma$, the primary signal is absent. Now we can get the detection probability. According to the literature [7], $P_{d}$ can be expressed as

$$
P_{d}=1-Q\left(\frac{\frac{1}{\gamma}+\frac{\Upsilon_{L} S N R}{\gamma(S N R+1)}-1}{\sqrt{2 / N_{s}}}\right)
$$

Where $\alpha_{l}$ denotes the correlation degree of the signal and $\Upsilon_{L}$ denotes the relative intensity of the signal. Define $\alpha_{l}=E(m(n) \cdot m(n+l)) / E\left(m^{2}(n)\right)$ and define $\Upsilon_{L}=2 \cdot \sum_{l=1}^{L-1}(L-l) \cdot \alpha_{l} / L$. The formula (14) can be simplified as

$$
\begin{aligned}
P_{d} & =1-Q\left(\frac{\frac{1}{\gamma}+\frac{\Upsilon_{L} S N R}{\gamma(S N R+1)}-1}{\sqrt{2 / N_{s}}}\right) \\
& =1-Q\left(\frac{\frac{\left(1+\Upsilon_{L}\right) S N R+1}{\gamma(S N R+1)}-1}{\sqrt{2 / N_{s}}}\right)
\end{aligned}
$$

Let $\frac{\left(1+\Upsilon_{L}\right) S N R+1}{\gamma(S N R+1)}=x$, we know that $x>1$ and $\left(1+\Upsilon_{L}\right)>\gamma$, so that $x$ increases when SNR increases, $\mathrm{Q}$ function decreases, then $P_{d}$ increases.

The main steps of the proposed spectrum sensing algorithm are summarized as follows:

Step 1) Choose a smoothing factor $\mathrm{L}$ and the number of sampling points $N_{s}$. 
Step 2) Compute the threshold $\gamma$ according to $\gamma=\frac{\left.1+(L-1) \sqrt{\frac{2}{N_{s} \pi}}\right)}{1-Q^{-1}\left(P_{f a}\right) \sqrt{\frac{2}{N_{s}}}}$. The $\gamma$ should be chosen to meet the requirement for the probability of false alarm.

Step 3) Compute the autocorrelations of the received signal $\lambda(l), l=0,1, \ldots, L-1$, and form the sample covariance matrix.

Step 4) Compute $T_{1}=\frac{1}{L} \sum_{i=1}^{L} \sum_{j=1}^{L}\left|r_{i j}\right| \quad$ and $T_{2}=\frac{1}{L} \sum_{i=1}^{L}\left|r_{i i}\right|$, where $r_{i j}$ are the elements of the sample covariance matrix $R_{y}\left(N_{s}\right)$.

Step 5) Determine the presence of the signal based on $T_{1}, T_{2}$ and $\gamma$. If $T_{1} / T_{2}>\gamma$, the signal exists, otherwise, the signal is absent.

\section{Simulation and Discussion}

In this section, we will give some simulation results. the simulation uses digital TV signal data captured in Washington area, the data samples have been used as a signal source reference model for the IEEE802.22 document. The parameters[6] are as follows: The data rate of the signal is $10.762 \mathrm{MHz}$, sampling rate is $21.524476 \mathrm{M} / \mathrm{sec}$, the signal time is $25 \mathrm{~s}$. The channel is rayleigh channel, and we choose $N_{s}=40000$ and $L=14$. At the same time, the performance of energy detection is simulated as contrast. The threshold of energy detection algorithm is set up as [8]

$$
\gamma_{E D}=\left(Q^{-1}\left(P_{f a}\right) \cdot \sqrt{2 N}+N\right) \cdot \sigma_{\eta}^{2}
$$

Now we need to know the noise variance $\sigma_{\eta}^{2}$, if the noise uncertainty $\rho$ is taken into account, then $P_{d}$ can be expressed as

$$
\begin{aligned}
& \left.P_{d}=\min _{\bar{\sigma}_{\eta}^{2} \in(1 / \rho) \sigma_{\eta}^{2}, \rho \sigma_{\eta}^{2}} Q \mid \frac{\gamma-\left(\sigma_{s}^{2}+{\overline{\sigma_{\eta}}}^{2}\right)}{\sqrt{2 / N}\left(\sigma_{s}^{2}+\overline{\sigma_{\eta}^{2}}\right)}\right\rfloor \\
& =Q\left[\frac{\gamma-\left(S N R+\frac{1}{\rho}\right)}{\sqrt{2 / N}\left(S N R+\frac{1}{\rho}\right)}\right]
\end{aligned}
$$

we can see the uncertainty $\rho$ has an impact on $P_{d}$.

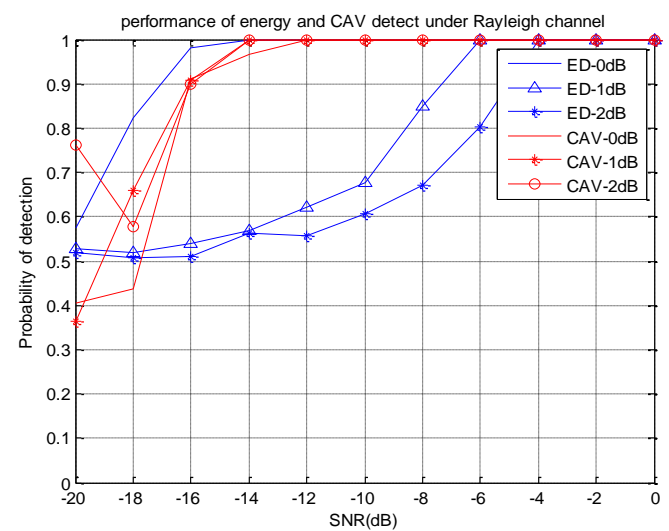

Fig. 1: Comparison of detection performance under different schemes

Fig. 1 illustates the detection probability versus SNR for the case that there exists noise uncertainty. We can see that the performance of the energy detection algorithm is very good when the noise is determined, but when the noise uncertainty is $1 \sim 2 \mathrm{~dB}$, the effect of energy detection probability is obvious. The larger noise uncertainty is, the larger impact on the energy detection algorithm. For CAV algorithm, there are some fluctuations when SNR is low. For example, when noise uncertainty is $2 \mathrm{~dB}, \mathrm{SNR}=-18 \mathrm{~dB}$, but it does not affect the overall judgment. Generally speaking, CAV algorithm has good robustness to noise uncertainty. 
The simulation results show the proposed of CAV algorithm offers an accurate performance when $S N R \geq-12 d B$.

In the practical communication, we will specify the reference range for $P_{f a}$.As mentioned earlier, according to IEEE802.22 document, we know that the reference range are given as $P_{f a}=0.01 \sim 0.1$, $P_{d}=0.9 \sim 0.99$. In order to compare the advantages and disadvantages of different detection schemes, Fig.2 illustrates the relationship between $P_{f a}$ and $P_{d}$. The range of $P_{f a}$ is $0.001 \sim 0.15$. When the noise uncertainty exists and $P_{f a} \geq 0.02$, We can see that $P_{d}$ in energy detection algorithm has little reaction to the change of $P_{f a}$. when the noise is certain, $P_{d}$ increases with the increase of $P_{f a}$. The $P_{d}$ of CAV algorithm also increases with the increase of $P_{f a}$.

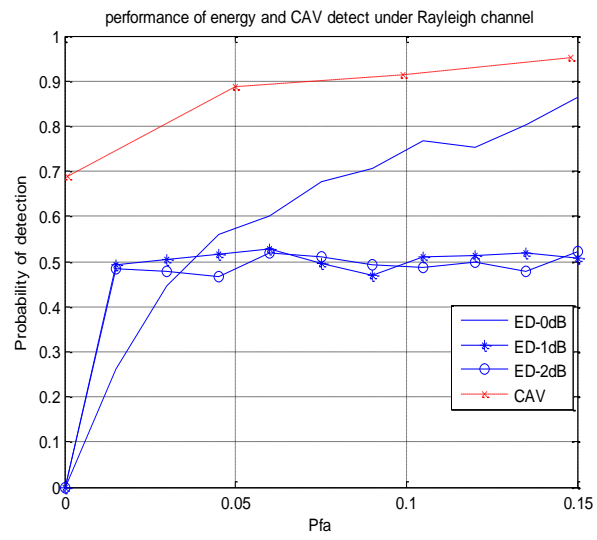

Fig.2: Comparison of detection performance under different $P_{f a}$

Table. 1 and Table. 2 illustrate the relationship between the threshold $\gamma 、 L$ and $N_{s}$ when $P_{f a}=0.05$ or $P_{f a}=0.1$. We can see that $P_{f a}$ is proportional to $\gamma, L$ is proportional to $\gamma, N_{s}$ is inversely proportional to $\gamma$, which is consistent with formula (13).

Table.1: the relationship between $L 、 N_{s}$ and $\gamma$ when $P_{f a}=0.05$

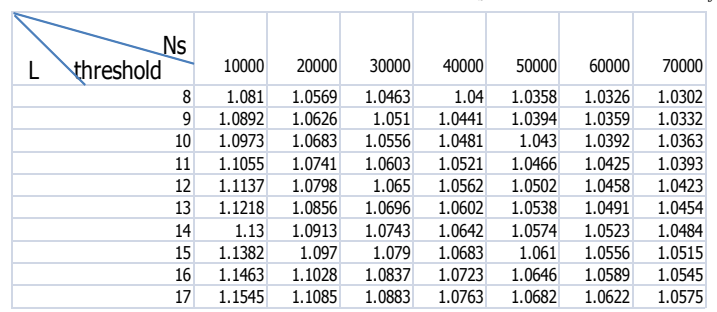

Table.2: the relationship between $L 、 N_{s}$ and $\gamma$ when $P_{f a}=0.1$

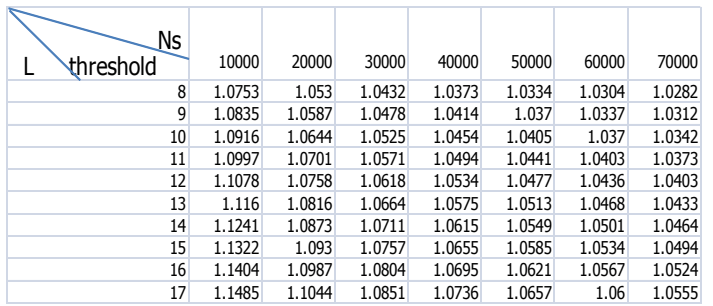

In order to guarantee the performance of CAV algorithm, we need to choose the values of $L$ and $N_{s}$. Fig.3 and Fig.4 illustrate the the relationship between $L 、 N_{s}$ and $P_{d}$. We can know that CAV algorithm offers an accurate performance when $L \geq 10, N_{s} \geq 4 \times 10^{4}$.

Fig.5 illustrates the relations between the theoretical value and DTV signal simulation results. We can see that they are consistent. There are some differences between them because in the calculation of $P_{d}$, in order to simplify calculation, some approximations are made and produce errors. However, the overall trend is consistent with the simulation of DTV signal. 


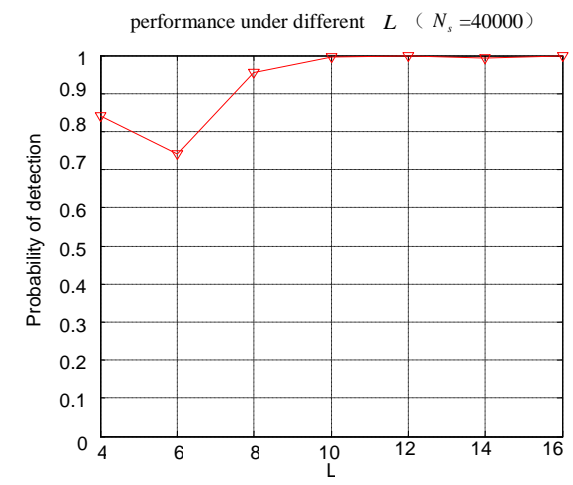

Fig.3: Performance under different $L \quad\left(N_{s}=40000\right)$

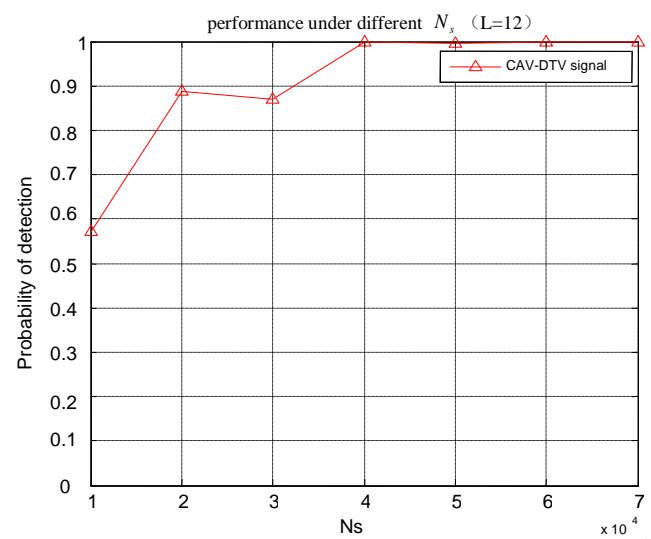

Fig.4: Performance under different $N_{s}(\mathrm{~L}=12)$

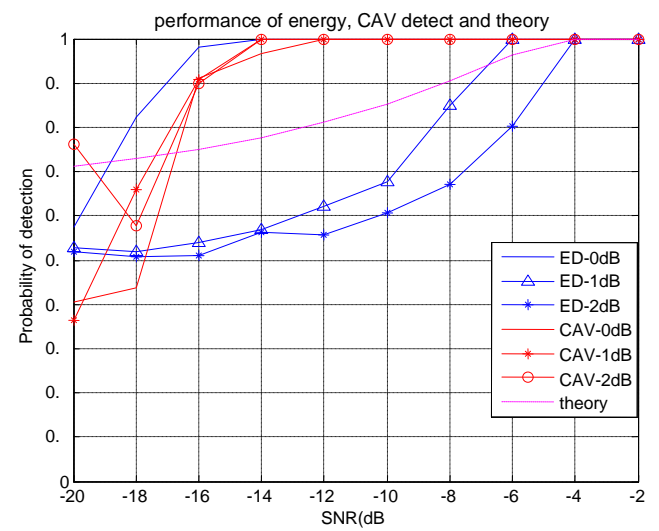

Fig.5: the relationship between SNR and $P_{d}$ in theoretical value and DTV signal simulation

In table 1 and table 2 , we set $\mathrm{L}=14$ and $N_{s}=4 \times 10^{4}$, when the range of $P_{f a}$ is $0 \sim 0.15$, then the range of $\gamma$ is $1.0601 \sim 1.0754$ ( $P_{f a}$ increases, $\gamma$ decreases). Analysis the relationship between $P_{d}$ and $\gamma$, we obtained that when $\gamma$ increases, the $P_{d}$ decreases. The simulation results are shown in Fig.6.

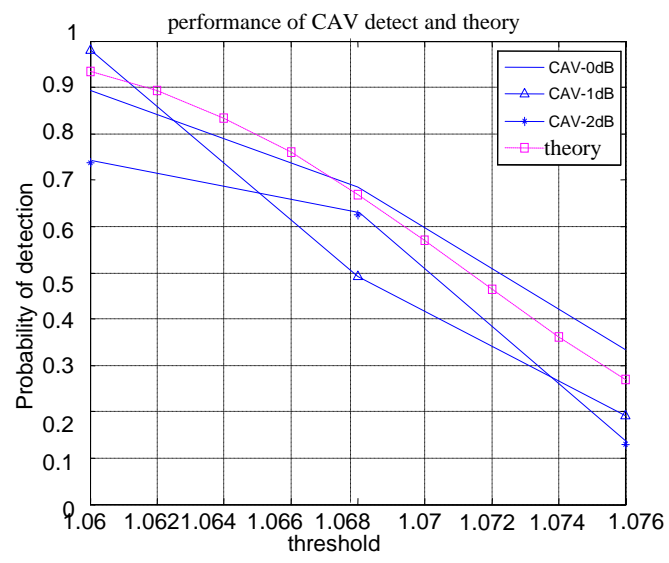

Fig.6: the relationship between $\gamma$ and $P_{d}$ in theoretical value and DTV signal simulation

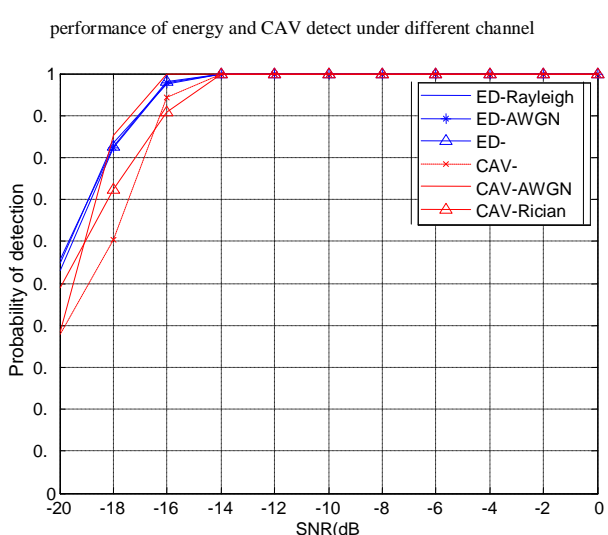

Fig.7: Energy detection and performance of CAV algorithm under different channels

Fig.7 illustrates the impact of channels to the detection performance. The common channels are Gauss white noise channel, rayleigh fading channel and rician fading Channel. In rician fading Channel, the energy ratio of reflection and scattering is 10 . We can see that the CAV algorithm has good adaptability to different channels. 


\section{Conclusions}

In this paper, sensing algorithms based on the sample signal autocorrelation have been discussed. The threshold are set by use of statistical theories, and then we can get the probability of detection. Throuth the simulations, we can clearly see the relations of differenct parameters, such as the smoothing factor $L$ 、 the number of available samples $N_{s}$ and the threshold $\gamma$, which can help us to choose the proper parameter values. Simulations based on DTV signals show that the proposed algorithm is better than energy detection when there exists noise uncertainty.

\section{Acknowledgements}

This paper was supported by the National Natural Science Foundation of China (Grant No.61561017) and Natural Science Foundation of Hainan Province, China(Grant No.20166214).

\section{References}

[1] CABRIC D, MISHRA S M, BRODERSEN R W. Implementation issues in spectrum sensing for cognitive radios[C]. The Thirty-Eighth Asilomar Conference, New York,USA: IEEE, 2004:772-776.

[2] SAHAI A, CABRIC D. Spectrum sensing: fundamental limits and practical challenges[C]. In Proc. IEEE International Symposium on New Frontiers in Dynamic Spectrum Access Networks(DySPAN), New York,USA: IEEE, 2005.

[3] GARDNER W A. Exploitation of spectral redundancy in cyclostationary signals[J]. IEEE Signal Processing Magazine, 1991, 8(2): 14-36.

[4] Zhe Sun, Weijia Han, Zan Li, Yan Zhang, and Meilu Lin. Spectrum Sensing Method without the Impact of Noise Uncertainty, global conference on signal and information processing,2013:1166-1169.

[5] R.Tandra and A.Sahai. SNR walls for feature detectors [J]. Dy SPAN 2007, The 2nd IEEE International Symposium on New Frontiers in Dynamic Spectrum Access Networks, Apr 2007:559-570.

[6] Yahong Rosa Zheng and Chengshan Xiao. Simulation Models with Correct Statistical Properties for Rayleigh Fading Channels [J]. IEEE Transactions on Communications, June, 2003, 51(6):920-929.

[7] ZENG Y H, LIANG Y-C. Spectrum-Sensing Algorithms for Cognitive Radio Based on Statistical Covariances [J]. IEEE Transactions on Vehicular Technology, 2009,58(4):1804-1815.

[8] Zeng Yonghong, Liang Yingchang. Maximum mini-mum eigenvalue detection for cognitive radio [C]// The 18th Annual IEEE International Symposium on Personal, Indoor and Mobile Radio Communications, 2007:1-5.

[9] Yonghong Zeng, Y C Liang. Spectrum sensing algorithm for cognitive radio based on statistical covariances [J]. IEEE Transactions on Vehicular Technology, 2009,58(4):1804-1815.

[10] Yonghong Zeng and Ying-Chang Liang. Reliability of Spectrum Sensing Under Noise and Interference Uncertainty [J]. IEEE International Conference on ICC Workshops, 2009:1-5.

[11] Bin Li, Yaobin Chen, Saisai Feng. Signal detection technology based on autocorrelation of signal sampling in Rayleigh channel [J]. Information and Electronic Engineering.Feb 2011,9(1):633-637

[12] J. Li, Y. Jiang, R. Fan. Recognition of Biological Signal Mixed Based on Wavelet Analysis. In: Y. Jiang, et al (eds.). Proc. of UK-China Sports Engineering Workshop. Liverpool: World Academic Union. 2007, pp. 1-8. (Use "References" Style)

[13] R. Dewri, and N. Chakraborti. Simulating recrystallization through cellular automata and genetic algorithms. Modelling Simul. Mater. Sci. Eng. 2005, 13 (3): 173-183.

[14] A. Gray. Modern Differential Geometry. CRE Press, 1998. 